\title{
A SUBADDITIVITY FORMULA FOR MULTIPLIER IDEALS ASSOCIATED TO LOG PAIRS
}

\author{
SHUNSUKE TAKAGI
}

(Communicated by Lev Borisov)

\begin{abstract}
As a generalization of formulas given in earlier papers by DemaillyEin-Lazarsfeld, Eisenstein and Takagi, we prove a subadditivity formula for multiplier ideals associated to log pairs.
\end{abstract}

\section{INTRODUCTION}

Multiplier ideals satisfy vanishing theorems, making them a fundamental tool in higher-dimensional algebraic geometry. They are defined as follows: let $(X, \Delta)$ be a $\log$ pair; that is, let $\Delta$ be an effective $\mathbb{Q}$-divisor on a normal variety $X$ over a field of characteristic zero such that $K_{X}+\Delta$ is $\mathbb{Q}$-Cartier. Let $\mathfrak{a} \subseteq \mathcal{O}_{X}$ be an ideal sheaf and $t>0$ be a real number. Suppose that $\pi: \widetilde{X} \rightarrow X$ is a log resolution of $(X, \Delta, \mathfrak{a})$; that is, $\pi$ is a proper birational morphism with $\widetilde{X}$ nonsingular such that $\mathfrak{a} \mathcal{O}_{\widetilde{X}}=\mathcal{O}_{\widetilde{X}}(-F)$ is invertible and $\operatorname{Exc}(\pi) \cup \operatorname{Supp}\left(\pi_{*}^{-1} \Delta\right) \cup \operatorname{Supp}(F)$ is a simple normal crossing divisor. Then the multiplier ideal $\mathcal{J}\left((X, \Delta) ; \mathfrak{a}^{t}\right)$ of $\mathfrak{a}$ with exponent $t$ for the pair $(X, \Delta)$ is

$$
\mathcal{J}\left((X, \Delta) ; \mathfrak{a}^{t}\right)=\pi_{*} \mathcal{O}_{\widetilde{X}}\left(\left\lceil K_{\widetilde{X}}-\pi^{*}\left(K_{X}+\Delta\right)-t F\right\rceil\right) \subseteq \mathcal{O}_{X}
$$

Demailly, Ein and Lazarsfeld [4] formulated a subadditivity property of multiplier ideals on nonsingular varieties, which states that the multiplier ideal of the product of two ideal sheaves is contained in the product of their individual multiplier ideals. Their formula has many interesting applications in algebraic geometry and commutative algebra, such as Fujita's approximation theorem (see [8] and 13 , Theorem 10.3.5]) and its local analogue (see [6]), a problem on the growth of symbolic powers of ideals in regular rings (see [5]), etc. Later, Takagi [18 and Eisenstein [7. generalized their formula to the case of $\mathbb{Q}$-Gorenstein varieties, that is, the case when $\Delta=0$ in the above definition of multiplier ideals. In this article, we study a further generalization to the case of log pairs, when the importance of multiplier ideals is particularly highlighted. The following is our main result.

Theorem (Theorems 2.3 and 3.5). Let $X$ be a normal variety over an algebraically closed field of characteristic zero and $\Delta$ be an effective $\mathbb{Q}$-divisor on $X$ such that $r\left(K_{X}+\Delta\right)$ is Cartier for some integer $r \geq 1$. Let $\mathrm{Jac}_{X}$ denote the Jacobian ideal sheaf of $X$. Then

$$
\operatorname{Jac}_{X} \cdot \mathcal{J}\left((X, \Delta) ; \mathfrak{a}^{s} \mathfrak{b}^{t} \mathcal{O}_{X}(-r \Delta)^{1 / r}\right) \subseteq \mathcal{J}\left((X, \Delta) ; \mathfrak{a}^{s}\right) \mathcal{J}\left((X, \Delta) ; \mathfrak{b}^{t}\right)
$$

for any ideal sheaves $\mathfrak{a}, \mathfrak{b} \subseteq \mathcal{O}_{X}$ and for any real numbers $s, t>0$.

Received by the editors June 9, 2011.

2010 Mathematics Subject Classification. Primary 14F18; Secondary 13A35, 14B05, 14E15. 
We give two proofs of this. The first proof is a refinement of the argument in [18. We give a subadditivity formula for big generalized test ideals, which itself is interesting from the point of view of algebraic geometry and commutative algebra in positive characteristic. Then we use a correspondence between multiplier ideals and big generalized test ideals (see [10] and [17]) to obtain the assertion. In the second proof, we employ the same method as that used in [7. We pull back the problem to the product $X \times X$ and then the desired formula on $X$ is obtained by restricting to the diagonal. We use a factorizing embedded resolution to compute the restriction of a multiplier ideal on $X \times X$ to the diagonal.

An interesting application of our formula is found in [2].

\section{Preliminaries on BIG Generalized test ideAlS}

In this section, we briefly review the definition and basic properties of big generalized test ideals, which we will need later. The reader is referred to [9], 10, 17] and 1 for details. The reader interested only in an algebro-geometric proof of our result can skip this section and go directly to Section 3 .

Throughout this paper, all schemes are Noetherian, excellent and separated. A graded family of ideal sheaves $\mathfrak{a}_{\bullet}=\left\{\mathfrak{a}_{m}\right\}_{m \geq 0}$ on an integral scheme $X$ means a collection of nonzero ideal sheaves $\mathfrak{a}_{m} \subseteq \mathcal{O}_{X}$, satisfying $\mathfrak{a}_{0}=\mathcal{O}_{X}$ and $\mathfrak{a}_{k} \mathfrak{a}_{l} \subseteq \mathfrak{a}_{k+l}$ for all $k, l \geq 1$. For example, given an ideal sheaf $\mathfrak{a} \subseteq \mathcal{O}_{X}$ and a real number $t \geq 0$, $\mathfrak{a}_{\bullet}=\left\{\mathfrak{a}^{\lceil t m\rceil}\right\}$ is a graded family of ideal sheaves on $X$. Another example of graded families of ideal sheaves is $I_{\Delta}^{(\bullet)}=\left\{\mathcal{O}_{X}(-\lceil m \Delta\rceil)\right\}$, where $\Delta$ is an effective $\mathbb{Q}$-divisor on a normal scheme $X$.

Let $X$ be an integral scheme of prime characteristic $p$. For each integer $e \geq 1$, we denote by $F^{e}: X \rightarrow X$ or $F^{e}: \mathcal{O}_{X} \rightarrow F_{*}^{e} \mathcal{O}_{X}$ the $e$-th iteration of the absolute Frobenius morphism on $X$. We say that $X$ is $F$-finite if $F: X \rightarrow X$ is a finite morphism. For example, a field $K$ of characteristic $p>0$ is $F$-finite if and only if $\left[K: K^{p}\right]$ is finite. Given an ideal sheaf $I \subseteq \mathcal{O}_{X}$, for each $q=p^{e}$, we denote by $I^{[q]} \subseteq \mathcal{O}_{X}$ the ideal sheaf identified with $I \cdot F_{*}^{e} \mathcal{O}_{X}$ via the identification $F_{*}^{e} \mathcal{O}_{X} \cong$ $\mathcal{O}_{X}$.

We give the definition of big generalized test ideals, using a generalization of tight closure [10], [17]. First we recall the definition of a generalization of tight closure.

Definition 1.1 ([10, Definition 6.1], [17, Definition 2.1], [16, Definition 2.16]). Let $X$ be a $d$-dimensional $F$-finite normal integral affine scheme of characteristic $p>0$, $\Delta$ be an effective $\mathbb{Q}$-divisor on $X$ and $\mathfrak{a}_{\bullet}$ be a graded family of ideals on $X$.

(i) Let $I \subseteq \mathcal{O}_{X}$ be a nonzero ideal. Then the $\left(\Delta, \mathfrak{a}_{\bullet}\right)$-tight closure $I^{*\left(\Delta, \mathfrak{a}_{\bullet}\right)}$ of $I$ is defined to be the ideal of $\mathcal{O}_{X}$ consisting of all $z \in \mathcal{O}_{X}$ for which there exists a nonzero element $c \in \mathcal{O}_{X}$ such that

$$
c \mathfrak{a}_{q-1} z^{q} \in I^{[q]} \mathcal{O}_{X}(\lceil(q-1) \Delta\rceil)
$$

for all large $q=p^{e}$.

(ii) Denote by $E=\bigoplus_{x} H_{x}^{d}\left(\omega_{X}\right)$ the direct sum, taken over all closed points $x \in X$, of the $d$-th local cohomology modules of the canonical module $\omega_{X}$ of $X$ with support on $x$. For each integer $e \geq 1$, let

$$
F_{E}^{e}: E=\bigoplus_{x} H_{x}^{d}\left(\mathcal{O}_{X}\left(K_{X}\right)\right) \rightarrow \bigoplus_{x} H_{x}^{d}\left(\mathcal{O}_{X}\left(p^{e} K_{X}\right)\right)
$$


be the map induced by the $e$-times iterated Frobenius map $F^{e}: \mathcal{O}_{X} \rightarrow$ $F_{*}^{e} \mathcal{O}_{X}$. Then the $\left(\Delta, \mathfrak{a}_{\bullet}\right)$-tight closure $0_{E}^{*\left(\Delta, \mathfrak{a}_{\bullet}\right)}$ of the zero submodule in $E$ is defined to be the submodule of $E$ consisting of all $z \in E$ for which there exists a nonzero element $c \in \mathcal{O}_{X}$ such that

$$
c \mathfrak{a}_{q-1} F_{E}^{e}(z)=0 \text { in } \bigoplus_{x} H_{x}^{d}\left(\mathcal{O}_{X}\left(q K_{X}+\lceil(q-1) \Delta\rceil\right)\right)
$$

for all large $q=p^{e}$.

(iii) We say that a nonzero element $c \in \mathcal{O}_{X}$ is a big sharp test element for the triple $\left(X, \Delta, \mathfrak{a}_{\bullet}\right)$ if for all $z \in 0_{E}^{*\left(\Delta, \mathfrak{a}^{t}\right)}$, we have

$$
c \mathfrak{a}_{q-1} F_{E}^{e}(z)=0 \text { in } \bigoplus_{x} H_{x}^{d}\left(\mathcal{O}_{X}\left(q K_{X}+\lceil(q-1) \Delta\rceil\right)\right)
$$

for every $q=p^{e}$. Big sharp test elements always exist (see [16, Lemma 2.17]).

Proposition-Definition 1.2 ([1, Definition-Proposition 3.3]; cf. [9, Lemma 2.1]). Let the notation be the same as in Definition 1.1. Then each of the following conditions defines the same ideal, which is called the big generalized test ideal for the triple $\left(X, \Delta, \mathfrak{a}_{\bullet}\right)$ and denoted by $\tau_{b}\left(X, \Delta, \mathfrak{a}_{\bullet}\right)$.

(a) $\operatorname{Ann}_{\mathcal{O}_{X}} 0_{E}^{*\left(\Delta, \mathfrak{a}_{\bullet}\right)}$.

(b) The ideal generated by all big sharp test elements for $\left(X, \Delta, \mathfrak{a}_{\bullet}\right)$.

(c) For any integer $e_{0} \geq 1$, the sum

$$
\sum_{e \geq e_{0}} \sum_{\phi_{e}} \phi_{e}\left(F_{*}^{e}\left(c \mathfrak{a}_{p^{e}-1}\right)\right)
$$

where $\phi_{e}$ ranges over all elements of $\operatorname{Hom}_{\mathcal{O}_{X}}\left(F_{*}^{e} \mathcal{O}_{X}\left(\left\lceil\left(p^{e}-1\right) \Delta\right\rceil\right), \mathcal{O}_{X}\right)$ and $c$ is a big sharp test element for $\left(X, \Delta, \mathfrak{a}_{\bullet}\right)$.

When $\Delta=0$, we denote this ideal simply by $\tau_{b}\left(X, \mathfrak{a}_{\bullet}\right)$. When $\mathfrak{a}_{\bullet}=\left\{\mathfrak{a}^{\lceil t m\rceil}\right\}$ for a nonzero ideal $\mathfrak{a} \subseteq \mathcal{O}_{X}$ and a real number $t>0$, we denote this ideal by $\tau_{b}\left(X, \Delta, \mathfrak{a}^{t}\right)$.

Remark 1.3. (1) Given graded families of ideals $\mathfrak{a}_{1, \bullet}, \ldots, \mathfrak{a}_{r, \bullet}$ on $X$, we can define the ideal $\tau_{b}\left(X, \Delta, \mathfrak{a}_{1, \bullet} \cdots \mathfrak{a}_{r, \bullet}\right)$ in the same manner as above.

(2) (19, Remark 1.4]) $\tau_{b}\left(X, \Delta, \mathfrak{a}_{\bullet}\right)$ is equal to the unique maximal element among the set of ideals $\left\{\tau_{b}\left(X, \Delta, \mathfrak{a}_{p^{e}}^{1 / p^{e}}\right)\right\}_{e \geq 0}$ with respect to inclusion. If $\mathfrak{a}_{\bullet}$ is a descending filtration, then $\tau_{b}\left(X, \Delta, \mathfrak{a}_{\bullet}\right)$ is equal to the unique maximal element among the set of ideals $\left\{\tau_{b}\left(X, \Delta, \mathfrak{a}_{m}^{1 / m}\right)\right\}$.

(3) Since the formation of $\tau_{b}\left(X, \Delta, \mathfrak{a}_{\bullet}\right)$ commutes with localization (see [9, Proposition 3.1]), we can define the ideal sheaf $\tau_{b}\left(X, \Delta, \mathfrak{a}_{\bullet}\right)$ when $X$ is a nonaffine scheme by gluing over affine charts.

Hara-Yoshida [10] and Takagi [17] proved a correspondence between multiplier ideals and big generalized test ideals. In order to state their results, we briefly recall how to reduce things from characteristic zero to characteristic $p>0$. We refer the reader to [11, Chapter 2] and [15, Section 3.2] for details.

Let $\Delta$ be an effective $\mathbb{Q}$-divisor on a normal variety $X$ over a field $k$ of characteristic zero. Let $\mathfrak{a} \subseteq \mathcal{O}_{X}$ be an ideal sheaf and $t>0$ be a real number. Then a model of $(X, \Delta, \mathfrak{a})$ over a finitely generated $\mathbb{Z}$-subalgebra $A$ of $k$ is a triple $\left(X_{A}, \Delta_{A}, \mathfrak{a}_{A}\right)$ of a normal integral scheme $X_{A}$ of finite type over $A$, an effective $\mathbb{Q}$-divisor $\Delta_{A}$ on $X_{A}$ and an ideal sheaf $\mathfrak{a}_{A} \subseteq \mathcal{O}_{X_{A}}$ such that $X_{A} \times_{\text {Spec } A} \operatorname{Spec} k \cong X, \rho^{*} \Delta_{A}=\Delta$ 
and $\rho^{-1} \mathfrak{a}_{A}=\mathfrak{a}$, where $\rho: X \rightarrow X_{A}$ is a natural projection. Given a closed point $\mu \in \operatorname{Spec} A$, we denote by $X_{\mu}$ (resp., $\Delta_{\mu}, \mathfrak{a}_{\mu}$ ) the fiber of $X_{A}$ (resp., $\Delta_{A}, \mathfrak{a}_{A}$ ) over $\mu$. Note that $X_{\mu}$ is a scheme of finite type over the residue field $\kappa(\mu)$ of $\mu$, which is a finite field.

Theorem 1.4 ([17, Theorem 3.2], [10, Theorem 6.8]). Let $X$ be a normal variety over a field $k$ of characteristic zero and $\Delta$ be an effective $\mathbb{Q}$-divisor on $X$ such that $K_{X}+\Delta$ is $\mathbb{Q}$-Cartier. Let $\mathfrak{a}$ be a nonzero ideal sheaf on $X$ and $t>0$ be a real number. Given any model $\left(X_{A}, \Delta_{A}, \mathfrak{a}_{A}\right)$ over a finitely generated $\mathbb{Z}$-subalgebra $A$ of $k$, there exists an open subset $W \subseteq$ Spec $A$ (depending on $t$ ) such that

$$
\mathcal{J}\left((X, \Delta) ; \mathfrak{a}^{t}\right)_{\mu}=\tau_{b}\left(X_{\mu}, \Delta_{\mu}, \mathfrak{a}_{\mu}^{t}\right)
$$

for every closed point $\mu \in W$.

\section{A PRoof Using BIG GENERALIZED TEST IDEALS}

In this section, we will give a subadditivity formula for multiplier ideals associated to log pairs, using big generalized test ideals. We start with the following lemma.

Lemma 2.1. Let $X$ be an $F$-finite normal integral affine scheme of characteristic $p>0$ and $\Delta$ be an effective $\mathbb{Q}$-divisor on $X$. Let $\mathfrak{a}, \mathfrak{b} \subseteq \mathcal{O}_{X}$ be ideals and $s, t>0$ be real numbers.

(1) For each integer $e \geq 1$,

$$
\tau_{b}\left(X, \mathfrak{a}^{s}\right) F_{*}^{e}\left(\mathcal{O}_{X}\left(-\left\lceil p^{e} \Delta\right\rceil\right)\right) \subseteq \tau_{b}\left(X, \Delta, \mathfrak{a}^{s}\right) F_{*}^{e} \mathcal{O}_{X}
$$

(2) Let $\mathcal{I}_{\Delta}^{(\bullet)}=\left\{\mathcal{O}_{X}(-\lceil m \Delta\rceil)\right\}$ be the graded family of ideals associated to $\Delta$. Then one has

$$
\tau_{b}\left(X, \mathfrak{a}^{s}\right)^{* \mathfrak{a}^{s}} \tau_{b}\left(X, \Delta, \mathfrak{a}^{s} \mathfrak{b}^{t} \mathcal{I}_{X}^{(\bullet)}\right) \subseteq \tau_{b}\left(X, \Delta, \mathfrak{a}^{s}\right) \tau_{b}\left(X, \Delta, \mathfrak{b}^{t}\right)
$$

Proof. (1) Let $c \in \mathcal{O}_{X}$ be a big sharp test element for both $\left(X, \mathfrak{a}^{t}\right)$ and $\left(X, \Delta, \mathfrak{a}^{t}\right)$. By Proposition-Definition 1.2

$$
\begin{aligned}
& \tau_{b}\left(X, \mathfrak{a}^{s}\right) F_{*}^{e}\left(\mathcal{O}_{X}\left(-\left\lceil p^{e} \Delta\right\rceil\right)\right) \\
= & \sum_{e^{\prime} \geq e} \sum_{\phi_{e^{\prime}}} F_{*}^{e}\left(\mathcal{O}_{X}\left(-\left\lceil p^{e} \Delta\right\rceil\right)\right) \phi_{e^{\prime}}\left(F_{*}^{e^{\prime}}\left(c \mathfrak{a}^{\left\lceil t\left(p^{e^{\prime}}-1\right)\right\rceil}\right)\right),
\end{aligned}
$$

where $\phi_{e^{\prime}}$ ranges over all elements of $\operatorname{Hom}_{\mathcal{O}_{X}}\left(F_{*}^{e^{\prime}} \mathcal{O}_{X}, \mathcal{O}_{X}\right)$. For all elements $s \in$ $\mathcal{O}_{X}\left(-\left\lceil p^{e} \Delta\right\rceil\right)$, since $s^{p^{e^{\prime}-e}} \in \mathcal{O}_{X}\left(-\left\lceil p^{e^{\prime}} \Delta\right\rceil\right)$,

$$
F_{*}^{e} s \cdot \phi_{e^{\prime}}: F_{*}^{e^{\prime}} \mathcal{O}_{X}\left(\left\lceil\left(p^{e^{\prime}}-1\right) \Delta\right\rceil\right) \stackrel{F_{*}^{e} s}{\longrightarrow} F_{*}^{e^{\prime}} \mathcal{O}_{X} \stackrel{\phi_{e^{\prime}}}{\longrightarrow} \mathcal{O}_{X}
$$

is viewed as an element of $\operatorname{Hom}_{\mathcal{O}_{X}}\left(F_{*}^{e^{\prime}} \mathcal{O}_{X}\left(\left\lceil\left(p^{e^{\prime}}-1\right) \Delta\right\rceil\right), \mathcal{O}_{X}\right)$. Thus, applying Proposition-Definition 1.2 again, one has

$$
\begin{aligned}
& \sum_{e^{\prime} \geq e} \sum_{\phi_{e^{\prime}}} F_{*}^{e}\left(\mathcal{O}_{X}\left(-\left\lceil p^{e} \Delta\right\rceil\right)\right) \phi_{e^{\prime}}\left(F_{*}^{e^{\prime}}\left(c \mathfrak{a}^{\left\lceil t\left(p^{e^{\prime}}-1\right)\right\rceil}\right)\right) \\
\subseteq & \sum_{e^{\prime} \geq e} \sum_{e^{\prime}} \psi_{e^{\prime}}\left(F_{*}^{e^{\prime}}\left(c \mathfrak{a}^{\left\lceil t\left(p^{e^{\prime}}-1\right)\right\rceil}\right)\right) \\
= & \tau_{b}\left(X, \Delta, \mathfrak{a}^{t}\right)
\end{aligned}
$$

where $\psi_{e^{\prime}}$ ranges over all elements of $\operatorname{Hom}_{\mathcal{O}_{X}}\left(F_{*}^{e^{\prime}} \mathcal{O}_{X}\left(\left\lceil\left(p^{e^{\prime}}-1\right) \Delta\right\rceil\right), \mathcal{O}_{X}\right)$. 
(2) Since the formation of big generalized test ideals commutes with localization and completion (see [9, Propositions 3.1 and 3.2]), we may assume that $(X, x)=$ Spec $R$, where $(R, \mathfrak{m})$ is a $d$-dimensional complete normal local ring of characteristic $p>0$. Let $E=H_{x}^{d}\left(\omega_{X}\right)$ be the $d$-th local cohomology module of $\omega_{X}$ with support on $x$ and let $F_{E}^{e}: E=H_{x}^{d}\left(\mathcal{O}_{X}\left(K_{X}\right)\right) \rightarrow H_{x}^{d}\left(\mathcal{O}_{X}\left(p^{e} K_{X}\right)\right)$ be the map induced by the $e$-times iterated Frobenius map $F^{e}: \mathcal{O}_{X} \rightarrow F_{*}^{e} \mathcal{O}_{X}$. Then by local duality, the assertion is equivalent to saying that

$$
\left(0_{E}^{*\left(\Delta, \mathfrak{a}^{s} \mathfrak{b}^{t} \mathcal{I}_{\Delta}^{(\bullet)}\right)}: \tau_{b}\left(X, \mathfrak{a}^{s}\right)^{* \mathfrak{a}^{s}}\right)_{E} \supseteq\left(0_{E}^{*\left(\Delta, \mathfrak{b}^{t}\right)}: \tau_{b}\left(X, \Delta, \mathfrak{a}^{s}\right)\right)_{E}
$$

Let $z \in\left(0_{E}^{*\left(\Delta, \mathfrak{b}^{t}\right)}: \tau_{b}\left(R, \Delta, \mathfrak{a}^{s}\right)\right)_{E}$. Then there exists a nonzero element $c \in \mathcal{O}_{X}$ such that

$$
c \mathfrak{b}^{\lceil t(q-1)\rceil} \tau_{b}\left(X, \Delta, \mathfrak{a}^{s}\right)^{[q]} F_{E}^{e}(z)=0 \text { in } H_{x}^{d}\left(\mathcal{O}_{X}\left(q K_{X}+\lceil(q-1) \Delta\rceil\right)\right)
$$

for all large $q=p^{e}$. Fix any nonzero element $\delta \in \mathcal{O}_{X}(-\lceil\Delta\rceil)$. By the definition of $\mathfrak{a}^{s}$-tight closure and (1), there exists another nonzero element $c^{\prime} \in \mathcal{O}_{X}$ such that

$$
\begin{aligned}
c^{\prime} \delta \mathfrak{a}^{\lceil s} & (q-1)\rceil \mathcal{O}_{X}(-\lceil(q-1) \Delta\rceil)\left(\tau_{b}\left(X, \mathfrak{a}^{s}\right)^{* \mathfrak{a}^{s}}\right)^{[q]} \\
& \subseteq c^{\prime} \mathfrak{a}^{\lceil s(q-1)\rceil} \mathcal{O}_{X}(-\lceil q \Delta\rceil)\left(\tau_{b}\left(X, \mathfrak{a}^{s}\right)^{* \mathfrak{a}^{s}}\right)^{[q]} \\
& \subseteq \mathcal{O}_{X}(-\lceil q \Delta\rceil) \tau_{b}\left(X, \mathfrak{a}^{s}\right)^{[q]} \\
& \subseteq \tau_{b}\left(X, \Delta, \mathfrak{a}^{s}\right)^{[q]}
\end{aligned}
$$

for all large $q=p^{e}$. Therefore, one has

$$
c c^{\prime} \delta \mathfrak{a}^{\lceil s(q-1)\rceil} \mathfrak{b}^{\lceil t(q-1)\rceil} \mathcal{O}_{X}(-\lceil(q-1) \Delta\rceil)\left(\tau_{b}\left(X, \mathfrak{a}^{s}\right)^{* \mathfrak{a}^{s}}\right)^{[q]} F_{E}^{e}(z)=0
$$

in $H_{x}^{d}\left(\mathcal{O}_{X}\left(q K_{X}+\lceil(q-1) \Delta\rceil\right)\right)$ for all large $q=p^{e}$. That is, $\tau_{b}\left(X, \mathfrak{a}^{s}\right)^{* \mathfrak{a}^{s}} z \subseteq$ $0_{E}^{*\left(\Delta, \mathfrak{a}^{s} \mathfrak{b}^{t}, \mathcal{I}_{\Delta}^{(\bullet)}\right)}$.

As a consequence of the above lemma, we obtain a subadditivity formula for big generalized test ideals. We stress that $K_{X}+\Delta$ is not necessarily $\mathbb{Q}$-Cartier in Proposition 2.2

Proposition 2.2. Let $X$ be a normal integral scheme essentially of finite type over an F-finite field and $\Delta$ be an effective $\mathbb{Q}$-divisor on $X$. Let $\mathcal{I}_{\Delta}^{(\bullet)}=\left\{\mathcal{O}_{X}(-\lceil m \Delta\rceil)\right\}$ denote the graded family of ideal sheaves associated to $\Delta$ and $\mathrm{Jac}_{X}$ denote the Jacobian ideal sheaf of $X$. Then

$$
\operatorname{Jac}_{X} \cdot \tau_{b}\left(X, \Delta, \mathfrak{a}^{s} \mathfrak{b}^{t} \mathcal{I}_{\Delta}^{(\bullet)}\right) \subseteq \tau_{b}\left(X, \Delta, \mathfrak{a}^{s}\right) \tau_{b}\left(X, \Delta, \mathfrak{b}^{t}\right)
$$

for any ideal sheaves $\mathfrak{a}, \mathfrak{b} \subseteq \mathcal{O}_{X}$ and for any real numbers $s, t>0$.

Proof. The question is local, so we may assume that $X$ is affine. Since $\operatorname{Jac}_{X} \subseteq$ $\tau_{b}\left(X, \mathfrak{a}^{s}\right)^{* \mathfrak{a}^{s}}$ by [18, Lemma 2.6], the assertion immediately follows from Lemma 2.1 $(2)$.

Before formulating a subadditivity property of multiplier ideals, we recall the definition of asymptotic multiplier ideal sheaves. Let $\Delta$ be an effective $\mathbb{Q}$-divisor on a normal variety $X$ over a field of characteristic zero such that $K_{X}+\Delta$ is $\mathbb{Q}$-Cartier. Let $a_{\bullet}=\left\{\mathfrak{a}_{m}\right\}$ be a graded family of ideal sheaves on $X$. Then the asymptotic multiplier ideal sheaf $\mathcal{J}\left((X, \Delta) ; \mathfrak{a}_{\bullet}\right)$ of $\mathfrak{a}_{\bullet}$ for the pair $(X, \Delta)$ is defined to be the 
unique maximal member among the family of ideal sheaves $\left\{\mathcal{J}\left((X, \Delta) ; \mathfrak{a}_{m}^{1 / m}\right)\right\}$ with respect to inclusion. We refer the reader to [13, Chapter 10] for details.

Theorem 2.3. Let $X$ be a normal variety over a field of characteristic zero and $\Delta$ be an effective $\mathbb{Q}$-divisor on $X$ such that $K_{X}+\Delta$ is $\mathbb{Q}$-Cartier. Let $\mathcal{I}_{\Delta}^{(\bullet)}=$ $\left\{\mathcal{O}_{X}(-\lceil m \Delta\rceil)\right\}$ denote the graded family of ideal sheaves associated to $\Delta$ and let $\mathrm{Jac}_{X}$ denote the Jacobian ideal sheaf of $X$. Then

$$
\operatorname{Jac}_{X} \cdot \mathcal{J}\left((X, \Delta) ; \mathfrak{a}^{s} \mathfrak{b}^{t} \mathcal{I}_{\Delta}^{(\bullet)}\right) \subseteq \mathcal{J}\left((X, \Delta) ; \mathfrak{a}^{s}\right) \mathcal{J}\left((X, \Delta) ; \mathfrak{b}^{t}\right)
$$

for any ideal sheaves $\mathfrak{a}, \mathfrak{b} \subseteq \mathcal{O}_{X}$ and for any real numbers $s, t>0$.

Proof. Take sufficiently large and divisible $m$ such that

$$
\mathcal{J}\left((X, \Delta) ; \mathfrak{a}^{s} \mathfrak{b}^{t} \mathcal{I}_{\Delta}^{(\bullet)}\right)=\mathcal{J}\left((X, \Delta) ; \mathfrak{a}^{s} \mathfrak{b}^{t} \mathcal{O}_{X}(-\lceil m \Delta\rceil)^{1 / m}\right)
$$

It follows from a combination of Remark 1.3, Theorem 1.4 and Proposition 2.2 that for a model $\left(X_{A}, \Delta_{A}, \mathfrak{a}_{A}, \mathfrak{b}_{A}\right)$ over a finitely generated $\mathbb{Z}$-subalgebra $A$ of $k$, there exists an open subset $W \subseteq \operatorname{Spec} A$ such that

$$
\begin{aligned}
& \left(\operatorname{Jac}_{X}\right)_{\mu} \cdot \mathcal{J}\left((X, \Delta) ; \mathfrak{a}^{s} \mathfrak{b}^{t} \mathcal{O}_{X}(-\lceil m \Delta\rceil)^{1 / m}\right)_{\mu} \\
& \quad=\operatorname{Jac}_{X_{\mu}} \cdot \tau_{b}\left(X_{\mu}, \Delta_{\mu}, \mathfrak{a}_{\mu}^{s} \mathfrak{b}_{\mu}^{t} \mathcal{O}_{X_{\mu}}\left(-\left\lceil m \Delta_{\mu}\right\rceil\right)^{1 / m}\right) \\
& \quad \subseteq \operatorname{Jac}_{X_{\mu}} \cdot \tau_{b}\left(X_{\mu}, \Delta_{\mu}, \mathfrak{a}_{\mu}^{s} \mathfrak{b}_{\mu}^{t} \mathcal{I}_{\Delta}^{(\bullet)}\right) \\
& \quad \subseteq \tau_{b}\left(X_{\mu}, \Delta_{\mu}, \mathfrak{a}_{\mu}^{s}\right) \cdot \tau_{b}\left(X_{\mu}, \Delta_{\mu}, \mathfrak{b}_{\mu}^{t}\right) \\
& \quad=\mathcal{J}\left((X, \Delta) ; \mathfrak{a}^{s}\right)_{\mu} \cdot \mathcal{J}\left((X, \Delta) ; \mathfrak{b}^{t}\right)_{\mu}
\end{aligned}
$$

for all closed points $\mu \in W$. This implies that

$$
\operatorname{Jac}_{X} \cdot \mathcal{J}\left((X, \Delta) ; \mathfrak{a}^{s} \mathfrak{b}^{t} \mathcal{O}_{X}(-\lceil m \Delta\rceil)^{1 / m}\right) \subseteq \mathcal{J}\left((X, \Delta) ; \mathfrak{a}^{s}\right) \mathcal{J}\left((X, \Delta) ; \mathfrak{b}^{t}\right) .
$$

\section{An AlgEBro-GeOMEtric PROOF}

In this section, employing the same method as that used in [7, we give an algebro-geometric proof of the above subadditivity formula for multiplier ideals. Throughout this section, let $X$ be a $d$-dimensional normal variety over an algebraically closed field of characteristic zero and $\Delta$ be an effective $\mathbb{Q}$-divisor on $X$ such that $K_{X}+\Delta$ is $\mathbb{Q}$-Cartier. For a closed subscheme $Z$ of $X$, we denote by $\mathcal{I}_{Z} \subseteq \mathcal{O}_{X}$ the defining ideal sheaf of $Z$ in $X$.

First we recall the definition of factorizing embedded resolutions.

Definition 3.1. Let $Z$ be a reduced closed subscheme of $X$ which is not contained in the singular locus $\operatorname{Sing}(X)$ of $X$. A factorizing embedded resolution of $Z$ in $X$ is a proper birational morphism $f: \bar{X} \rightarrow X$ with $\bar{X}$ smooth such that

(a) $f$ is an isomorphism at every generic point of $Z \subseteq X$,

(b) the exceptional locus $\operatorname{Exc}(f)$ is a simple normal crossing divisor,

(c) the strict transform $\bar{Z}$ of $Z$ in $\bar{X}$ is smooth and has simple normal crossings with $\operatorname{Exc}(f)$,

(d) $\mathcal{I}_{Z} \mathcal{O}_{\bar{X}}=\mathcal{I}_{\bar{Z}} \mathcal{O}_{\bar{X}}\left(-R_{Z}\right)$, where $R_{Z}$ is an $f$-exceptional divisor on $\bar{X}$.

Such a resolution always exists (see [3]). 
Lemma 3.2 (cf. [7, Lemma 3.6]). Let $\mathfrak{a} \subseteq \mathcal{O}_{X}$ be an ideal sheaf and $t>0$ be a real number. Let $Z \subseteq X$ be a reduced equidimensional closed subscheme of codimension $c$, none of whose components is contained in $\operatorname{Sing}(X) \cup \operatorname{Supp}(\Delta) \cup \operatorname{Supp}(V(\mathfrak{a}))$. Let $f: \bar{X} \rightarrow X$ be a log resolution of $(X, \Delta, \mathfrak{a})$ which is simultaneously a factorizing embedded resolution of $Z$ in $X$ so that $\mathcal{I}_{Z} \mathcal{O}_{\bar{X}}=\mathcal{I}_{\bar{Z}} \mathcal{O}_{\bar{X}}\left(-R_{Z}\right)$, where $\bar{Z}$ is the strict transform of $Z$ in $\bar{X}$. Put

$$
B:=\left\lceil K_{\bar{X}}-f^{*}\left(X_{X}+\Delta\right)-t \cdot f^{-1}(V(\mathfrak{a}))-c \cdot R_{Z}\right\rceil .
$$

Then the restriction map

$$
\left.f_{*} \mathcal{O}_{\bar{X}}(B) \rightarrow f\right|_{\bar{Z} *} \mathcal{O}_{\bar{Z}}\left(\left.B\right|_{\bar{Z}}\right)
$$

is surjective.

Proof. It suffices to show that $R^{1} f_{*}\left(\mathcal{I}_{\bar{Z}} \mathcal{O}_{\bar{X}}(B)\right)=0$. Let $g: Y \rightarrow \bar{X}$ be the blowup of $\bar{X}$ along $\bar{Z}$ with reduced exceptional divisor $E$, and denote by $h=(g \circ f): Y \rightarrow X$ the composite morphism. Since

$$
\begin{aligned}
\left(\mathcal{I}_{\bar{Z}} \mathcal{O}_{Y}\right) g^{*} \mathcal{O}_{\bar{X}}(B) & =\mathcal{O}_{Y}\left(\left\lceil g^{*} K_{\bar{X}}-h^{*}\left(K_{X}+\Delta\right)-t \cdot h^{-1}(V(\mathfrak{a}))-c \cdot g^{*} R_{Z}-E\right\rceil\right) \\
& =\mathcal{O}_{Y}\left(\left\lceil K_{Y}-h^{*}\left(K_{X}+\Delta\right)-t \cdot h^{-1}(V(\mathfrak{a}))-c \cdot h^{-1}(X)\right\rceil\right),
\end{aligned}
$$

it follows from the Kawamata-Viehweg vanishing theorem that

$$
R^{i} h_{*}\left(\left(\mathcal{I}_{\bar{Z}} \mathcal{O}_{Y}\right) g^{*} \mathcal{O}_{\bar{X}}(B)\right)=R^{i} g_{*}\left(\left(\mathcal{I}_{\bar{Z}} \mathcal{O}_{Y}\right) g^{*} \mathcal{O}_{\bar{X}}(B)\right)=0
$$

for all $i>0$. We use the Leray spectral sequence to conclude that

$$
R^{i} f_{*}\left(\mathcal{I}_{\bar{Z}} \mathcal{O}_{\bar{X}}(B)\right)=R^{i} f_{*}\left(g_{*}\left(\left(\mathcal{I}_{\bar{Z}} \mathcal{O}_{Y}\right) g^{*} \mathcal{O}_{\bar{X}}(B)\right)\right)=0
$$

for all $i>0$.

Definition 3.3. Given any positive integer $r$ such that $r\left(K_{X}+\Delta\right)$ is Cartier, consider the natural map

$$
\rho_{r, \Delta}:\left(\Omega_{X}^{d}\right)^{\otimes r} \rightarrow \mathcal{O}_{X}\left(r K_{X}\right) \rightarrow \mathcal{O}_{X}\left(r\left(K_{X}+\Delta\right)\right) .
$$

Let $\mathcal{I}_{r, \Delta} \subseteq \mathcal{O}_{X}$ be the ideal sheaf so that $\operatorname{Im} \rho_{r, \Delta}=\mathcal{I}_{r, \Delta} \mathcal{O}_{X}\left(r\left(K_{X}+\Delta\right)\right)$. Note that if $\operatorname{Jac}_{X}$ is the Jacobian ideal sheaf of $X$, then $\operatorname{Jac}_{X}^{r} \cdot \mathcal{O}_{X}(-r \Delta) \subseteq \mathcal{I}_{r, \Delta}$.

Lemma 3.4 (cf. [7, Lemma 4.5]). Let $f: \bar{X} \rightarrow X$ be a birational morphism with $X$ smooth and $\mathrm{Jac}_{f}$ be the Jacobian ideal sheaf of $f$. Given an integer $r \geq 1$ such that $r\left(K_{X}+\Delta\right)$ is Cartier, one has

$$
\operatorname{Jac}_{f}^{r}=\mathcal{I}_{r, \Delta} \mathcal{O}_{\bar{X}}\left(-r\left(K_{\bar{X}}-f^{*}\left(K_{X}+\Delta\right)\right)\right) .
$$

Proof. First note that by the definition of $\mathrm{Jac}_{f}$, the image of the natural map $f^{*}\left(\Omega_{X}^{d}\right)^{\otimes r} \rightarrow \mathcal{O}_{\bar{X}}\left(r K_{\bar{X}}\right)$ coincides with $\operatorname{Jac}_{f}^{r} \cdot \mathcal{O}_{\bar{X}}\left(r K_{\bar{X}}\right)$. Consider the decomposition $r\left(K_{\bar{X}}-f^{*}\left(K_{X}+\Delta\right)\right)=K_{+}-K_{-}$, where $K_{+}, K_{-}$are effective divisors on $\bar{X}$. Then we have the following commutative diagram:

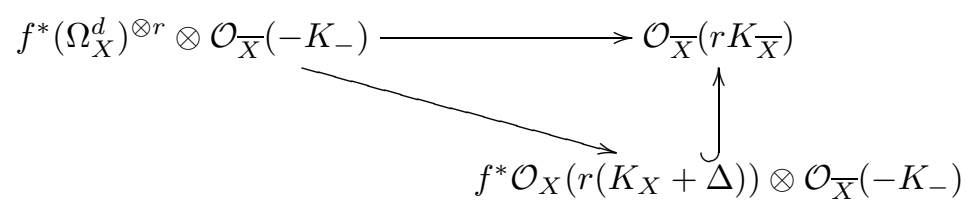


Computing the images of these maps, we see that

$$
\operatorname{Jac}_{f}^{r} \cdot \mathcal{O}_{\bar{X}}\left(-K_{-}\right)=\mathcal{I}_{r, \Delta} \mathcal{O}_{\bar{X}}\left(-K_{+}\right),
$$

which gives the assertion.

Now we state a subadditivity formula for multiplier ideals involving the ideal sheaf $\mathcal{I}_{r, \Delta}$.

Theorem 3.5. Let $X$ be a normal variety over an algebraically closed field of characteristic zero and $\Delta$ be an effective $\mathbb{Q}$-divisor on $X$ such that $r\left(K_{X}+\Delta\right)$ is Cartier for an integer $r \geq 1$. Let $\mathcal{I}_{r, \Delta}$ be the ideal sheaf given in Definition 3.3 . Then

$$
\mathcal{J}\left((X, \Delta) ; \mathfrak{a}^{s} \mathfrak{b}^{t} \mathcal{I}_{r, \Delta}^{1 / r}\right) \subseteq \mathcal{J}\left((X, \Delta) ; \mathfrak{a}^{s}\right) \mathcal{J}\left((X, \Delta) ; \mathfrak{b}^{t}\right)
$$

for any ideal sheaves $\mathfrak{a}, \mathfrak{b} \subseteq \mathcal{O}_{X}$ and for any real numbers $s, t>0$. In particular,

$$
\overline{\operatorname{Jac}_{X}} \cdot \mathcal{J}\left((X, \Delta) ; \mathfrak{a}^{s} \mathfrak{b}^{t} \mathcal{I}_{X}^{(\bullet)}\right) \subseteq \mathcal{J}\left((X, \Delta) ; \mathfrak{a}^{s}\right) \mathcal{J}\left((X, \Delta) ; \mathfrak{b}^{t}\right),
$$

where $\overline{\operatorname{Jac}_{X}}$ is the integral closure of the Jacobian ideal sheaf of $X$ and $\mathcal{I}_{\Delta}^{(\bullet)}=$ $\left\{\mathcal{O}_{X}(-\lceil m \Delta\rceil)\right\}$ is the graded family of ideal sheaves associated to $\Delta$.

Proof. Let $p_{1}, p_{2}: X \times X \rightarrow X$ be the natural projections. We regard $X$ as a closed subvariety of $X \times X$ via the diagonal embedding $X \hookrightarrow X \times X$. Since

$$
J\left((X, \Delta) ; \mathfrak{a}^{s}\right) \mathcal{J}\left((X, \Delta) ; \mathfrak{b}^{t}\right)=\left.\mathcal{J}\left(\left(X \times X, p_{1}^{*} \Delta+p_{2}^{*} \Delta\right) ;\left(p_{1}^{-1} \mathfrak{a}\right)^{s}\left(p_{2}^{-1} \mathfrak{b}\right)^{t}\right)\right|_{X},
$$

it is enough to show that

$$
\left.\mathcal{J}\left((X, \Delta) ; \mathfrak{a}^{s} \mathfrak{b}^{t} \mathcal{I}_{r, \Delta}^{1 / r}\right) \subseteq \mathcal{J}\left(\left(X \times X, p_{1}^{*} \Delta+p_{2}^{*} \Delta\right) ;\left(p_{1}^{-1} \mathfrak{a}\right)^{s}\left(p_{2}^{-1} \mathfrak{b}\right)^{t}\right)\right|_{X} .
$$

Let $\pi: \widetilde{X} \rightarrow X$ be a $\log$ resolution of $\Delta, \mathfrak{a}, \mathfrak{b}$ and $\mathcal{I}_{r, \Delta}$ so that $\mathcal{I}_{r, \Delta} \mathcal{O}_{\widetilde{X}}=\mathcal{O}_{\widetilde{X}}\left(-F_{r, \Delta}\right)$, and denote by $g=\pi \times \pi: \tilde{X} \times \tilde{X} \rightarrow X \times X$ the product morphism. Note that the restriction of $g$ to the diagonal is nothing but $\pi$. Let $h: Y \rightarrow \widetilde{X} \times \widetilde{X}$ be a morphism such that the composite morphism $f=(h \circ g): Y \rightarrow X \times X$ is a factoring embedded resolution of $X$ in $X \times X$ and $\mathcal{I}_{X} \mathcal{O}_{Y}=\mathcal{I}_{\bar{X}} \mathcal{O}_{Y}\left(-R_{X}\right)$, where $\bar{X}$ is the strict transform of $X$ in $Y$ :

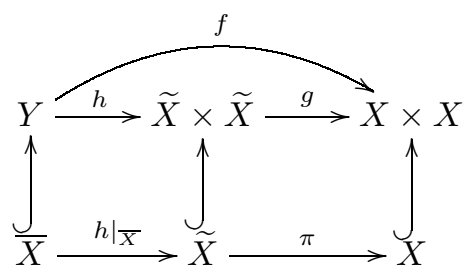

Put $B=K_{Y}-f^{*}\left(K_{X \times X}+p_{1}^{*} \Delta+p_{2}^{*} \Delta\right)$ and denote by $d$ the dimension of $X$. It then follows from Lemma 3.2 that

$$
\begin{aligned}
\left.f\right|_{\bar{X}_{*}} & \mathcal{O}_{\bar{X}}\left(\left.\left\lceil B-s \cdot f^{-1}\left(V\left(p_{1}^{-1} \mathfrak{a}\right)\right)-t \cdot f^{-1}\left(V\left(p_{2}^{-1} \mathfrak{b}\right)\right)-d \cdot R_{X}\right\rceil\right|_{X}\right) \\
& =\left.f_{*} \mathcal{O}_{Y}\left(\left\lceil B-s \cdot f^{-1}\left(V\left(p_{1}^{-1} \mathfrak{a}\right)\right)-t \cdot f^{-1}\left(V\left(p_{2}^{-1} \mathfrak{b}\right)\right)-d \cdot R_{X}\right\rceil\right)\right|_{X} \\
& \left.\subseteq f_{*} \mathcal{O}_{Y}\left(\left\lceil B-s \cdot f^{-1}\left(V\left(p_{1}^{-1} \mathfrak{a}\right)\right)-t \cdot f^{-1}\left(V\left(p_{2}^{-1} \mathfrak{b}\right)\right)\right\rceil\right)\right|_{X} \\
& =\left.\mathcal{J}\left(\left(X \times X, p_{1}^{*} \Delta+p_{2}^{*} \Delta\right) ;\left(p_{1}^{-1} \mathfrak{a}\right)^{s}\left(p_{2}^{-1} \mathfrak{b}\right)^{t}\right)\right|_{X}
\end{aligned}
$$


Claim.

$$
\left.\left(B-d \cdot R_{X}\right)\right|_{\bar{X}} \geq K_{\bar{X}}-\left.f\right|_{\bar{X}} ^{*}\left(K_{X}+\Delta\right)-\left.\frac{1}{r} \cdot h\right|_{\bar{X}} ^{*} F_{r, \Delta} \cdot
$$

Proof of Claim. Applying Lemma 3.4 to $h, \pi$ and $\left.f\right|_{\bar{X}}$, one has

$$
\begin{aligned}
\operatorname{Jac}_{h} & =\mathcal{O}_{Y}\left(-K_{Y / \widetilde{X} \times \widetilde{X}}\right), \\
\operatorname{Jac}_{\pi}^{r} & =\mathcal{O}_{\widetilde{X}}\left(-r\left(K_{\widetilde{X}}-\pi^{*}\left(K_{X}+\Delta\right)\right)-F_{r, \Delta}\right), \\
\operatorname{Jac}_{\left.f\right|_{\bar{X}}}^{r} & =\mathcal{O}_{\bar{X}}\left(-r\left(K_{\bar{X}}-\left.f\right|_{\bar{X}}{ }^{*}\left(K_{X}+\Delta\right)\right)-\left.h\right|_{\bar{X}} ^{*} F_{r, \Delta}\right) .
\end{aligned}
$$

It follows from [7, Lemma 6.3] ( $X$ is assumed to be normal and $\mathbb{Q}$-Gorenstein in [7, but the same statement holds when $X$ is only normal) that

$$
\left.\operatorname{Jac}_{h}\right|_{\bar{X}}\left(\operatorname{Jac}_{\pi} \cdot \mathcal{O}_{\bar{X}}\right)^{2} \subseteq \operatorname{Jac}_{\left.f\right|_{\bar{X}}} \cdot \mathcal{O}_{\bar{X}}\left(-\left.d \cdot R_{X}\right|_{\bar{X}}\right),
$$

which is equivalent to saying that

$$
\begin{aligned}
& -\left.K_{Y / \tilde{X} \times \tilde{X}}\right|_{\bar{X}}-\left.2 \cdot h\right|_{\bar{X}}{ }^{*} K_{\tilde{X}}+\left.2 \cdot f\right|_{\bar{X}}{ }^{*}\left(K_{X}+\Delta\right)-\left.\frac{2}{r} \cdot h\right|_{\bar{X}} ^{*} F_{r, \Delta} \\
\leq & -K_{\bar{X}}+\left.f\right|_{\bar{X}}{ }^{*}\left(K_{X}+\Delta\right)-\left.\frac{1}{r} \cdot h\right|_{\bar{X}} ^{*} F_{r, \Delta}-\left.d \cdot R_{X}\right|_{\bar{X}} .
\end{aligned}
$$

Note that $\left.\left(K_{\tilde{X} \times \tilde{X}}-g^{*}\left(K_{X \times X}+p_{1}^{*} \Delta+p_{2}^{*} \Delta\right)\right)\right|_{\tilde{X}}=2\left(K_{\tilde{X}}-\pi^{*}\left(K_{X}+\Delta\right)\right)$. Thus, substituting this equality to the above inequality, one has

$$
K_{\bar{X}}-\left.f\right|_{\bar{X}} ^{*}\left(K_{X}+\Delta\right)-\left.\frac{1}{r} \cdot h\right|_{\bar{X}} ^{*} F_{r, \Delta} \leq\left.\left(B-d \cdot R_{X}\right)\right|_{\bar{X}} .
$$

By the above claim, we have

$$
\begin{aligned}
& \mathcal{J}(\left.(X, \Delta) ; \mathfrak{a}^{s} \mathfrak{b}^{t} \mathcal{I}_{r, \Delta}^{1 / r}\right) \\
&=\left.f\right|_{\bar{X} *} \mathcal{O}_{\bar{X}}\left(\left\lceilK_{\bar{X}}-\left.f\right|_{\bar{X}}{ }^{*}\left(K_{X}+\Delta\right)-\left.s \cdot f\right|_{\bar{X}} ^{-1}(V(\mathfrak{a}))\right.\right. \\
&\left.\left.\quad-\left.t \cdot f\right|_{\bar{X}} ^{-1}(V(\mathfrak{b}))-\left.\frac{1}{r} \cdot h\right|_{\bar{X}} ^{*} F_{r, \Delta}\right\rceil\right) \\
&\left.\quad \subseteq f\right|_{\bar{X} *} \mathcal{O}_{\bar{X}}\left(\left.\left\lceil B-s \cdot f^{-1}\left(V\left(p_{1}^{-1} \mathfrak{a}\right)\right)-t \cdot f^{-1}\left(V\left(p_{2}^{-1} \mathfrak{b}\right)\right)-d \cdot R_{X}\right\rceil\right|_{\bar{X}}\right) \\
&\left.\quad \subseteq \mathcal{J}\left(\left(X \times X, p_{1}^{*} \Delta+p_{2}^{*} \Delta\right) ;\left(p_{1}^{-1} \mathfrak{a}\right)^{s}\left(p_{2}^{-1} \mathfrak{b}\right)^{t}\right)\right|_{X} \cdot
\end{aligned}
$$

Remark 3.6. The inclusion $(\star)$ in Theorem 3.5 involves not the Jacobian ideal sheaf but its integral closure, so Theorem 3.5 is a little bit stronger than Theorem 2.3 in this sense. We do not know at the moment how to prove the inclusion $(\star)$ using big generalized test ideals. The difficulty is illustrated in the fact that big generalized test ideals are not necessarily integrally closed (see [14]).

\section{ACKNOWLEDGEMENTS}

The author would like to thank Tommaso de Fernex, who raised this problem. $\mathrm{He}$ is indebted to Salvatore Cacciola for pointing out a mistake in a previous version of the article. The author also would like to express his gratitude to the Massachusetts Institute of Technology, where a part of this work was done, for their hospitality during the winter of 2010-2011. The author was partially supported by Grant-in-Aid for Young Scientists (B) 20740019 from JSPS and by the Program for Improvement of Research Environment for Young Researchers from SCF commissioned by MEXT of Japan. 


\section{REFERENCES}

[1] M. Blickle, K. Schwede, S. Takagi and W. Zhang, Discreteness and rationality of $F$-jumping numbers on singular varieties, Math. Ann. 347 (2010), 917-949. MR2658149

[2] S. Boucksom, T. de Fernex and C. Favre, The volume of an isolated singularity, http://arxiv. org/abs/1011.2847, to appear in Duke Math. J.

[3] A. Bravo, S. Encinas and O. Villamayor, A simplified proof of desingularization and applications, Rev. Mat. Iberoamericana 21 (2005), 349-458. MR2174912 (2006h:14018)

[4] J.-P. Demailly, L. Ein and R. Lazarsfeld, A subadditivity property of multiplier ideals, Michigan Math. J. 48 (2000), 137-156. MR.1786484 (2002a:14016)

[5] L. Ein, R. Lazarsfeld, and K. Smith, Uniform bounds and symbolic powers on smooth varieties, Invent. Math. 144 (2001), 241-252. MR1826369(2002b:13001)

[6] L. Ein, R. Lazarsfeld, and K. Smith, Uniform approximation of Abhyankar valuation ideals in smooth function fields, Amer. J. Math. 125 (2003), 409-440. MR1963690 (2003m:13004)

[7] E. Eisenstein, Generalization of the restriction theorem for multiplier ideals, arXiv:1001.2841, to appear in Ann. Inst. Fourier (Grenoble).

[8] T. Fujita, Approximating Zariski decomposition of big line bundles, Kodai Math. J. 17 (1994), 1-3. MR1262949 (95c:14053)

[9] N. Hara and S. Takagi, On a generalization of test ideals, Nagoya Math. J. 175 (2004), 59-74. MR2085311 (2005g:13009)

[10] N. Hara and K. Yoshida, A generalization of tight closure and multiplier ideals, Trans. Amer. Math. Soc. 355 (2003), 3143-3174. MR1974679(2004i:13003)

[11] M. Hochster and C. Huneke, Tight closure in equal characteristic zero, preprint.

[12] M. Kawakita, On a comparison of minimal log discrepancies in terms of motivic integration, J. Reine Angew. Math. 620 (2008), 55-65. MR2427975 (2010i:14021)

[13] R. Lazarsfeld, Positivity in Algebraic Geometry II, Ergeb. Math. Grenzgeb. 3. Folge, A Series of Modern Surveys in Mathematics, vol. 49, Springer-Verlag, Berlin, 2004. MR2095472 (2005k:14001b)

[14] M. McDermott, Test ideals in diagonal hypersurface rings. II, J. Algebra 264 (2003), 296-304. MR.1980699 (2004d:13004)

[15] M. Mustaţă and V. Srinivas, Ordinary varieties and the comparison between multiplier ideals and test ideals, Nagoya Math. J. 204 (2011), 125-157. MR2863367

[16] K. Schwede, Centers of F-purity, Math. Z. 265 (2010), 687-714. MR2644316 (2011e:13011)

[17] S. Takagi, An interpretation of multiplier ideals via tight closure, J. Algebraic Geom. 13 (2004), 393-415. MR2047704 (2005c:13002)

[18] S. Takagi, Formulas for multiplier ideals on singular varieties, Amer. J. Math. 128 (2006), no. 6, 1345-1362. MR2275023 (2007i:14006)

[19] S. Takagi and K. Yoshida, Generalized test ideals and symbolic powers, Michigan Math. J. 57 (2008), 711-724. MR2492477 (2010d:13007)

Department of Mathematics, Kyushu University, 744 Motooka, Nishi-Ku, Fukuoka 819-0395, JAPAN

E-mail address: stakagi@math.kyushu-u.ac.jp

Current address: Graduate School of Mathematical Sciences, University of Tokyo, 3-8-1

Komaba, Meguro-ku, Tokyo 153-8914, Japan

E-mail address: stakagi@ms.u-tokyo.ac.jp 